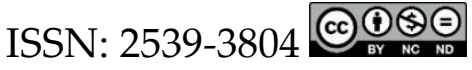

\title{
Premisas implícitas y relaciones de transitividad
}

Miguel López-Astorga

Universidad de Talca

Talca, Chile

\section{Resumen}

En 2010, Johnson-Laird propuso que el hecho de que no todas las proposiciones que establecen relaciones permiten interpretar tales relaciones como transitivas es un problema para ciertas teorías; en concreto, para aquellas que pretenden explicar el razonamiento y la comunicación humana sintácticamente. Su argumento es, básicamente, que si tales proposiciones hacen referencia a formas lógicas idénticas, las relaciones que debería ser posible derivar de ellas tendrían que ser también similares. No obstante, en este trabajo se defiende que las teorías sintácticas pueden responder a esta crítica. Es suficiente considerar la existencia de premisas implícitas que solo ejercen su acción en ciertos contextos. Así, se propondrá que solo algunas proposiciones correspondientes a relaciones permiten pensar en transitividad porque únicamente algunas de tales proposiciones pueden vincularse con premisas ocultas que expresen esa relación. Como también se mostrará, desde esta óptica se pueden comprender igualmente errores a este respecto.

Palabras clave: forma lógica; pragmática; premisas implícitas; relaciones transitivas; sintaxis.

\begin{abstract}
Implicit premises and transitivity relationships

In 2010, Johnson-Laird stated that the fact that not all of propositions establishing relationships allow for its interpretation in terms of transitivity is a problem for certain theories; particularly for those trying to explain reasoning and human communication syntactically. Ina nutshell, his argument is that if those propositions refer to identical logical forms, the relationships that could possibly be derived from them would have to be identical as well. However, in this paper, my claim is that syntactic theories can respond to this criticism. It is enough to
\end{abstract}


consider the existence of implicit premises that only act in certain contexts. Thus, it will be argued that only some propositions enable to think about transitivity because only some of such propositions can be linked to hidden premises expressing that relationship. As it will also be shown, from this perspective, mistakes in this regard can be understood in the same way.

Key words: logical form; pragmatics; implicit premises; transitive relationships; syntax.

\section{Résumé}

\section{Prémisses implicites et relations de transitivité}

En 2010, Johnson-Laird a déclaré que le fait que toutes les propositions fournissant établissant des relations ne permettent pas d'interpréter ces relations comme transitives est un problème pour certaines théories; en particulier pour celles qui tentent d'expliquer le raisonnement et la communication humaine syntaxiquement. Son argument est, fondamentalement, que si ces propositions se réfèrent à des formes logiques identiques, les relations qu'il devrait être possible d'en tirer devraient également être égales. Cependant, dans cet article, on soutient que les théories syntaxiques peuvent répondre à cette critique. Il suffit de considérer l'existence de prémisses implicites qui n'agissent que dans certains contextes. Ainsi, on prétendra que seules certaines propositions permettent de penser à la transitivité car seules certaines de ces propositions peuvent être liées à des prémisses cachées exprimant cette relation. Comme on le verra aussi, de ce point de vue, des erreurs à cet égard peuvent être comprises de la même manière.

Mots-clés: forme logique; pragmatique; prémisses implicites; relations transitives; syntaxe. 


\section{CóMO CITAR ESTE ARTÍCULO}

López-Astorga, M. (2019). Premisas implícitas y relaciones de transitividad. Lenguaje, 47(1), 73-90. doi: 10.25100/lenguaje.v47i1.7162 


\section{INTRODUCCIÓN}

El trabajo de Johnson-Laird (2010) constituyó, sin duda, una crítica difícil de soslayar contra la idea de que es posible vincular las oraciones o las sentencias con formas lógicas que, en principio, les corresponden. Es cierto, no obstante, que muchos de sus argumentos han sido ya respondidos (véase, p.ej., López-Astorga, 2015); pero uno de ellos, que va a ser analizado en estas páginas, a pesar de fundamentarse en bases muy sólidas, no parece haber recibido mucha atención. Tal argumento es el referente a las proposiciones que establecen relaciones y al hecho de que solo en algunos casos tales relaciones suelen ser consideradas como transitivas, siendo entendidas, en el resto de las situaciones, como intransitivas. El planteamiento de Johnson-Laird (2010) en este sentido es, indiscutiblemente, un problema para cualquier teoría que defienda tesis semejantes a la de la existencia de una sintaxis del pensamiento (p.ej., la teoría de la lógica mental tal y como está presentada en la literatura y en textos como los citados más abajo), las cuales suelen proponer que los individuos piensan recurriendo a esquemas similares, aunque no necesariamente idénticos, a los que proporciona la lógica estándar (entendemos aquí por estos últimos esquemas los que se pueden apreciar en trabajos como los de, p.ej., Deaño, 1999; Gentzen, 1934, 1935). Y esto puede ser fácilmente comprobado si prestamos atención al rol que efectivamente desempeñan las reglas formales en estas teorías.

Para, por poner el caso, la teoría de la lógica mental, la mera presencia de ciertas premisas con determinadas estructuras debe llevar necesariamente a conclusiones muy concretas. Dicho de otro modo, dadas ciertas estructuras formales, algunos esquemas lógicos se aplican de manera cuasi automática. Tal es el caso de la conocida regla de Modus Ponendo Ponens, que en dicha teoría recibe la denominación de 'Esquema Núcleo (Core Schema) 7' (Braine y O'Brien, 1998a, p. 80) y que puede expresarse formalmente así:

$$
p \rightarrow q, p \therefore q
$$

(Donde ' $\rightarrow$ ' expresa relación condicional y ' $\because$ ' indica que las fórmulas de la izquierda permiten derivar la de la derecha).

Como decimos, los defensores de la teoría de la lógica mental aseguran que, siempre que tenemos dos premisas con estructuras como las de $\mathrm{p} \rightarrow \mathrm{q}$ y $\mathrm{p}$, q se deduce forzosamente, y ello, por supuesto, con independencia del contenido semántico de p y q. Evidentemente, la maquinaria y el marco general de esta teoría 
son, en realidad, mucho más complejos, ya que diferencian entre distintos tipos de esquemas (por ejemplo, Esquemas Núcleo, como el indicado, que se aplican siempre que es posible, Esquemas Alimentadores -Feeder Schemata-, que solo son utilizados cuando ello puede llevar a nuevas deducciones, o Esquemas de Incompatibilidad -Incompatibility Schemata-, que revelan que existe una contradicción y que, por tanto, al menos uno de los supuestos de los que se parte no es correcto) y hablan de un programa de razonamiento que indica claramente cuándo y en qué orden exacto se aplican esos tipos de regla formal (véase, p.ej., Braine y O'Brien, 1998a). No obstante, el problema que observa Johnson-Laird (2010) es que, en el caso de las relaciones, ocurre un fenómeno inexplicable desde este marco general. Si suponemos que 'P $\alpha \beta$ ' significa que existe una relación entre $\alpha$ y $\beta$, tenemos que el hecho de que tal relación se interprete como transitiva o como intransitiva depende del contenido efectivo de $\mathrm{P}$, pues, según sea dicho contenido, la siguiente inferencia será considerada correcta o no por los individuos:

\section{[2] Pxy, Pyz $\therefore$ Pxz (para todo $x, y, z)$}

Empero, si es el contenido de P lo que posibilita entender la relación como transitiva o intransitiva, no podemos hablar de esquemas sintácticos, ya que la deducción de la conclusión se hace en este caso en virtud de criterios esencialmente semánticos (el significado de P). Sin embargo, aquí se va a argumentar que esto no es un problema real para las teorías formales. Desde ellas, se puede suponer que [2] nunca es válido y que, si, a veces, se puede inferir Pxz de Pxy y Pyz, ello se debe únicamente a la acción de ciertas premisas pragmáticas que pueden ser vinculadas a la relación concreta establecida por $\mathrm{P}$ en algunas ocasiones. Esto, como se explicará más abajo, no es incompatible con las tesis generales de las teorías sintácticas, las cuales suelen plantear la presencia de premisas pragmáticas en las inferencias, y permite pensar que, si bien la semántica interviene en cierta medida en el proceso, no es el único factor y que también se puede notar en él una clara dimensión sintáctica.

Para proceder en esta dirección, primero, se explicará más detenidamente en qué consiste exactamente la crítica que Johnson-Laird (2010) les hace a las teorías formales apoyándose en el problema de la transitividad de las relaciones. Así, en un segundo momento, se mostrará que la diferencia entre las conclusiones que, en ciertas circunstancias, se pueden obtener a partir de premisas que, a pesar de establecer relaciones distintas, mantienen una estructura formal idéntica, puede ser entendida asumiendo el rol que en el pensamiento y el lenguaje pueden 
desempeñar las premisas pragmáticas. Como hemos dicho, tal rol se halla explícitamente expuesto en la literatura especializada y las teorías formales o sintácticas suelen reconocerlo. No obstante, antes de mostrar este punto, empezamos, como también hemos indicado, con los argumentos de Johnson-Laird.

\section{EL PROBLEMA DE LA FORMA LÓGICA DE LAS RELACIONES}

Ya hemos avanzado que Johnson-Laird (2010) entiende que la dificultad de las teorías sintácticas, con respecto al tema que estamos abordando, es que la mayor parte de las relaciones que se expresan mediante sentencias en lenguaje natural responden a la misma estructura, esto es, a P $\alpha \beta$. Ciertamente, las formas lógicas de las premisas de estos dos ejemplos son las mismas:

[3] "Ann is taller than Beth.

Beth is taller than Cath.

Therefore, Ann is taller than Cath" (Johnson-Laird, 2010, p. 208).

[Ann tiene mayor estatura que Beth.

Beth tiene mayor estatura que Cath.

Por tanto, Ann tiene mayor estatura que Cath].

[4] "Ann is a blood relative of Beth.

Beth is a blood relative of Chris.

What follows?" (Johnson-Laird, 2010, p. 209).

[Ann es pariente de sangre de Beth.

Beth es pariente de sangre de Chris.

¿Qué se sigue?].

Tales formas lógicas son, obviamente, $\mathrm{Pab}$ y Pbc (donde ' $\mathrm{a}$ ' hace referencia a Ann, ' $b$ ' representa a Beth y 'c' significa 'Cath' en [3] y 'Chris' en [4]). No obstante, las conclusiones que se derivan en las dos inferencias no son, en principio, las mismas. Es evidente que la conclusión de [3] es la que aparece en ella, esto es, que 'Ann tiene mayor estatura que Cath', o, si se prefiere, Pac. En este sentido, Johnson-Laird (2010) nos recuerda que, como demostraron Bryant y Trabasso (1971), incluso niños de corta edad pueden llegar a tal conclusión. Sin embargo, la dificultad se revela en [4]. Como igualmente comenta Johnson-Laird (2010), en un estudio realizado por él mismo junto con Goodwin (Goodwin y Johnson-Laird, 2008), se obtuvo como resultado que muchas personas tienden a concluir, en esta 
última inferencia, que Ann es pariente de sangre de Chris, o, si se prefiere, también Pac, lo cual, en este caso, es incorrecto. El propio Johnson-Laird (2010) nos proporciona un contraejemplo: Ann puede ser la madre de Beth y Chris el padre de esta última, con lo que, aunque se cumple lo señalado en las premisas, Ann y Chris no son parientes de sangre. Esta tendencia al error, empero, en su opinión, se puede revertir. Es suficiente con ofrecerles a los individuos indicios claros de lo que realmente entraña el matrimonio en las relaciones de parentesco y recordarles lo que verdaderamente implica tal unión con respecto a la consanguinidad. Que esto es así fue probado también por Goodwin y Johnson-Laird (2008), trabajo en el que se puede apreciar que, cuando una inferencia como [4] se acompaña con información de esa índole, la tendencia en los resultados cambia, pero lo que nos interesa principalmente aquí es que, como se ha apuntado, esto plantea una dificultad importante a las teorías formales, pues refleja claramente que no basta con que las premisas tengan ciertas formas lógicas para derivar determinadas conclusiones.

Johnson-Laird (2010) revisa algunos enfoques que han tratado de solucionar el mencionado problema, como, por ejemplo, el de Politzer (2004) o el de Tsal (1977); pero, en general, argumenta contra ellos que, en realidad, no explican tampoco por qué las relaciones son consideradas transitivas en algunos casos y en otros no. De esta manera, lo que propone es abordar el fenómeno desde la teoría que él defiende, la teoría de los modelos mentales, un marco cognitivo vigente en nuestros días, esencialmente semántico, con un amplio alcance y de cuyas tesis fundamentales se puede encontrar una síntesis en trabajos relativamente recientes, como, por ejemplo, el de Johnson-Laird, Khemlani y Goodwin (2015). No obstante, esta última teoría no es relevante para los objetivos de este trabajo. De hecho, centrarnos en ella y analizarla en detalle nos alejaría considerablemente de nuestros fines aquí. Como se ha señalado, el foco fundamental de estas páginas va dirigido a mostrar que el problema de la transitividad no es un problema real para las teorías sintácticas y, de este modo, que los argumentos de Johnson-Laird (2010) no suponen una objeción insalvable para ellas. Por consiguiente, a tal foco nos vamos a ceñir, y lo vamos a hacer argumentando que, si bien, ciertamente las teorías sintácticas se centran principalmente, como su propio nombre indica, en la sintaxis, ello no significa que sean exclusivamente sintácticas. Son claramente compatibles también con la acción de elementos pragmáticos y, en muy diversas ocasiones, sus defensores apelan explícitamente a la presencia de premisas procedentes de nuestro conocimiento general y que influyen en nuestra actividad inferencial. Cómo son exactamente tales premisas y cómo pueden actuar cuando 
un individuo se enfrenta a relaciones diádicas como las descritas se muestra en el apartado siguiente.

\section{Las premisas pragmáticas en las teorías sintácticas y su acción en las relaciones diádicas}

Prácticamente, ninguna teoría formal o sintáctica propone que los seres humanos razonen en el vacío o ex nihilo y considerando únicamente las premisas que se mencionan de modo explícito en las inferencias. Para la mayor parte de estas teorías, defender una idea semejante sería apoyar una visión muy simple y pobre de la dinámica intelectual humana que ignoraría la auténtica riqueza de los procesos mentales reales. De este modo, suelen proponer que, además de a las premisas explícitas, los individuos suelen atender, de igual modo, a premisas implícitas que poseen en virtud de su conocimiento general y que la pragmática puede hacer patentes. Esto, evidentemente, revela que las teorías sintácticas no son, como se ha dicho, exclusivamente sintácticas. Sin embargo, si se revisa la literatura acerca de ellas, se puede notar fácilmente, de la misma manera, que lo cierto es que nunca han pretendido serlo, que siempre han tendido a considerar la presencia de premisas pragmáticas en el razonamiento (véase, p. ej., para el caso concreto de la teoría de la lógica mental, Braine y O'Brien, 1998b) y que, en diversos trabajos, han reconocido que no pretenden exigir exclusividad y que los procesos mentales sintácticos bien pueden estar complementados con otros de diverso tipo, entre ellos, por supuesto, los semánticos (véase, igualmente, para el caso particular de la teoría anterior, p.ej., O’Brien, 1998). Así, las teorías formales permiten entender lo que sucede en inferencias como la siguiente:

[5] Si bebo alcohol, entonces no puedo conducir.

Bebo cerveza.

La mayor parte de los individuos derivará, dadas estas premisas, evidentemente, que no puedo conducir. Empero, es obvio que, desde el punto de vista formal, falta información para poder hacer eso adecuadamente, y ello se puede notar tanto si se adopta un marco sintáctico basado en el cálculo proposicional como si se trabaja desde la lógica de predicados de primer orden. En el primer caso, la estructura formal de la inferencia sería: 
[6]

$$
\mathrm{p} \rightarrow \neg \mathrm{q}
$$

$\mathrm{R}$

(Donde ' $\neg$ ' significa negación y ' $\mathrm{p}$ ' hace referencia al hecho de que bebo alcohol, ' $q$ ' al hecho de que conduzco y ' $r$ ' al hecho de que bebo cerveza).

$\mathrm{E}$, indudablemente, nada se puede inferir en cálculo proposicional de solamente dos premisas como $\mathrm{p} \rightarrow \neg \mathrm{q}$ y $\mathrm{r}$.

Por otra parte, la situación no es muy diferente si recurrimos al cálculo de predicados de primer orden. En este caso, se puede pensar en fórmulas como estas:

[7] $\quad \forall x(\mathrm{Ax} \wedge \mathrm{Bax}) \rightarrow \neg \mathrm{Ca}$

Bac

(Donde ' $\forall$ ' es el cuantificador universal, ' $\wedge$ ' representa la conjunción lógica, 'A $\alpha$ ' indica que $\alpha$ es una bebida alcohólica, $B \alpha \beta$ describe la situación en la que $\alpha$ bebe $\beta$, 'C $\alpha$ ' alude al escenario en el que $\alpha$ conduce, ' $a$ ' hace referencia al hablante o emisor, esto es, a la primera persona del singular, $y$ ' $c$ ' significa 'cerveza').

Sin duda, también estamos aquí en un caso de nihil sequitur, es decir, en un caso en el que nada se sigue a partir de las premisas.

No obstante, si se admite la existencia de premisas implícitas como las mencionadas, se puede explicar la razón por la que se tiende a concluir que yo no puedo conducir, y ello tanto si formalizamos las premisas como en [6] como si lo hacemos como en [7]. Claramente, la premisa implícita de [6] es $\mathrm{r} \rightarrow \mathrm{p}$, la cual establece que, como se sabe por conocimiento general, si yo bebo cerveza, entonces yo bebo alcohol y permite esta deducción:
[8] 1. $\mathrm{p} \rightarrow \neg \mathrm{q}$
(premisa)
2. $r$
(premisa)
3. $r \rightarrow p$
(premisa implícita)
4. $\mathrm{p}$
(Modus Ponendo Ponens 2, 3)
5. $\neg \mathrm{q}$
(Modus Ponendo Ponens 1, 4) 
Por su parte, la premisa oculta en [7] sería Ac, la cual revela, como también es sabido por conocimiento general, que la cerveza es una bebida alcohólica y posibilita esta inferencia:

1. $\forall \mathrm{x}(\mathrm{Ax} \wedge \mathrm{Bax}) \rightarrow \neg \mathrm{Ca}$

2. Bac

3. Ac

4. Ac $\wedge$ Bac

5. $\mathrm{Ac} \wedge \mathrm{Bac} \rightarrow \neg \mathrm{Ca}$

6. ᄀCa (premisa)

(premisa)

(premisa implícita)

(Introducción de Conjunción 3, 2)

(Eliminación de Cuantificador Universal 1)

(la regla de Introducción de Conjunción es: $\mathrm{p}, \mathrm{q} \therefore \mathrm{p} \wedge \mathrm{q}$-véase, $\mathrm{p} . e j .$, Deaño, 1999- y la regla de Eliminación de Cuantificador Universal es: $\forall \mathrm{x} P \mathrm{Px} \therefore \mathrm{Pa}$, donde 'a' simboliza una constante -véase, p.ej., Deaño, 1999-).

[6] y [8] representarían la situación de un modo semejante a como se entendería en la teoría de la lógica mental tal y como está expresada, por ejemplo, en Braine y O'Brien (1998a). No obstante, [7] y [9] sirven para ilustrar que, si bien son posibles diversos enfoques formales o sintácticos, la acción de las premisas implícitas puede ser comprendida de un modo similar desde esos enfoques y permite solucionar los mismos problemas en ellos. Esto significa que, si desde esta perspectiva se pueden entender los fenómenos descritos por Johnson-Laird (2010) comentados más arriba y explicarlos, tales actividades se pueden realizar sea cual sea el enfoque formal que se adopte. Aquí se va a mostrar que, efectivamente, se pueden resolver las dificultades planteadas por Johnson-Laird (2010) recurriendo a la simbología de la lógica de predicados de primer orden, y ello, simplemente, porque vamos a revisar relaciones diádicas y parece más oportuno expresar tales relaciones con esa simbología. Sin embargo, como decimos y se podrá notar fácilmente tras nuestra argumentación (tan fácilmente que, para evitar caer en la trivialidad, no se darán mayores detalles al respecto), desde el lenguaje de otros marcos lógicos también podría exponerse fácilmente una explicación parecida.

No obstante, antes de proceder en tal dirección, parece oportuno atender a dos posibles objeciones que se podrían plantear contra lo propuesto en este apartado. Por una parte, se podría pensar que se están ignorando textos tan 
fundamentales para el análisis de las relaciones entre la sintaxis, la semántica y la pragmática como, por ejemplo, los de Gamut $(2002,2010)$ o el de Grice (1975). Empero, tales textos no han sido mencionados porque, más allá de las relaciones más o menos explícitas que se pueden establecer entre tales trabajos y las teorías formales, aquí se está intentando trabajar básicamente solo sobre lo argumentado explícitamente de manera habitual desde tales teorías. Por otra parte, se podría, del mismo modo, argumentar, con base en el ejemplo propuesto, que, en realidad, las premisas implícitas se hallan más vinculadas a la semántica que a la pragmática, ya que saber que la cerveza es una bebida alcohólica es algo que se deriva del propio significado de la palabra 'cerveza', independientemente del contexto pragmático en el que este término es utilizado. A pesar de ello, hablamos aquí de premisas pragmáticas porque, hasta donde entendemos las teorías formales, si bien las premisas implícitas derivan verdaderamente del conocimiento general y, por consiguiente, pueden tener una dimensión más semántica que pragmática, es el contexto el que hace que el individuo recurra a ellas para realizar sus inferencias. Así, premisas como la tercera tanto en [8] como en [9] son, efectivamente, producto de procesos semánticos. No obstante, se puede defender que solo se recurre a ellas si el contexto inferencial lo requiere, es decir, en virtud de factores pragmáticos.

\section{Algunos casos planteados por Johnson-Laird revisados desde la óptica de las teorías sintácticas}

Quizás lo primero que habría que plantear desde una óptica formal es que, si existe una sintaxis del pensamiento, [2] no es una regla que forme parte de ella. De hecho, dicha regla no es válida ni siquiera en la lógica estándar pues, como sabemos, en el cálculo de predicados de primer orden, no es siempre correcto que

$\forall \mathrm{xyz}(\mathrm{Pxy} \wedge \mathrm{Pyz} \rightarrow \mathrm{Pxz})$

Es cierto que todas las teorías formales no son iguales y que los esquemas sintácticos que admiten no coinciden exactamente en todos los casos con los de la lógica clásica. Sin embargo, hasta donde sabemos, [2], o, si se prefiere, [10], no es aceptada tampoco en ninguna de las teorías formales que se defienden en la literatura. Por consiguiente, se puede pensar, legítimamente, que no es un esquema de nuestra mente que forme parte de nuestra dotación intelectual natural y que, si recurrimos a menudo a estructuras similares, ello es, simplemente, porque disponemos de premisas pragmáticas muy parecidas en nuestro conocimiento general. Así, puede parecer un esquema de la sintaxis del pensamiento porque la 
pragmática nos conduce a su estructura con frecuencia, pero no lo es realmente, ya que es también la propia pragmática la que nos puede revelar que su conclusión, o su segunda cláusula, no es adecuada en todas las ocasiones. Ciertamente, son muchos los casos en los que [2], o [10], se cumple. Por ejemplo, esta es una premisa pragmática con la que solemos contar en nuestros razonamientos:

$$
\forall \mathrm{xyz}[(\mathrm{x}=\mathrm{y}) \wedge(\mathrm{y}=\mathrm{z}) \rightarrow(\mathrm{x}=\mathrm{z})]
$$

Efectivamente, cuando la relación es de identidad, y, de esta manera, $P \alpha \beta$ equivale a $\alpha=\beta$, la transitividad ocurre y el conocimiento general hace explícita una premisa como la que aparece en [11]. $\mathrm{Y}$ lo mismo sucede cuando ' $\mathrm{P}$ ' significa 'mayor que', esto es, cuando P $\alpha \beta$ equivale a $\alpha>\beta$, o 'menor que', esto es, cuando Po $\beta$ equivale a $\alpha<\beta$. En estos casos, también se da la transitividad y se pueden señalar premisas implícitas, respectivamente, las que figuran a continuación:

$$
\forall x y z[(x>y) \wedge(y>z) \rightarrow(x>z)]
$$

[13] $\forall x y z[(x<y) \wedge(y<z) \rightarrow(x<z)]$

De esta manera, recurriendo de nuevo a uno de los ejemplos de JohnsonLaird (2010), los defensores de una teoría formal podrían argumentar fácilmente que lo que sucede en [3] es que el contenido de las premisas permite vincular la inferencia con una fórmula como [12] y que, como evidenciaron Bryant y Trabasso (1971), se trata de una derivación simple hasta para niños muy pequeños porque permite, de una manera rápida y cuasi automática, una derivación como la que sigue:

[14] 1. $a>b$

2. $b>c$

3. $\forall x y z[(x>y) \wedge(y>z) \rightarrow(x>z)]$

4. $(\mathrm{a}>\mathrm{b}) \wedge(\mathrm{b}>\mathrm{c}) \rightarrow(\mathrm{a}>\mathrm{c})$ (premisa)

(premisa)

(premisa implícita)

(Eliminación de Cuantificador 
5. $(\mathrm{a}>\mathrm{b}) \wedge(\mathrm{b}>\mathrm{c}) \quad$ (Introducción de Conjunción 1, 2)

6. $(\mathrm{a}>\mathrm{c}) \quad$ (Modus Ponendo Ponens 4,5$)$

(Donde ' $a$ ' es Ann, ' $b$ ' es Beth, ' $c$ ' es Cath $y$ ' $>$ ' no significa exactamente 'mayor que', sino 'tiene mayor estatura que').

Empero, como se ha indicado, esto no es siempre así. Hay casos en los que el conocimiento general nos revela que, a diferencia de lo que sucede en [14], el consecuente de [10], que en [14] se torna en [12], tiene que estar negado o ser convertido en Pxz $\vee \neg \operatorname{Pxz}$ (donde ' $\vee$ ' expresa disyunción). El primer caso se puede observar en relaciones como, por ejemplo, la de ser el jefe inmediatamente superior. Obviamente, si a es el jefe inmediatamente superior de $b$ y $b$ es el jefe inmediatamente superior de c, a no puede ser el jefe inmediatamente superior de c, ya que lo es b. En esta circunstancia, es indudable que la premisa pragmática no sería [10], sino

$$
\forall \mathrm{xyz}(\mathrm{Pxy} \wedge \mathrm{Pyz} \rightarrow \neg \mathrm{Pxz})
$$

(Donde ' $\mathrm{P}$ ' hace referencia, como se ha dicho, a la relación de ser el jefe inmediatamente superior).

Por lo que se refiere al otro caso, a aquel en que es necesario introducir una disyunción en el consecuente, un buen ejemplo puede ser el de la relación de amistad. Sin duda, que a sea amigo de b y b sea amigo de $\mathrm{c}$ no significa que a sea amigo de c. Puede serlo y puede no serlo. Por tanto, de nuevo, la premisa implícita más apropiada aquí no parece ser [10], sino una semejante a esta:

$$
\forall \mathrm{xyz}(\mathrm{Pxy} \wedge \mathrm{Pyz} \rightarrow \mathrm{Pxz} \vee \neg \mathrm{Pxz})
$$

(Donde ' $\mathrm{P}$ ' hace ahora referencia, como también se ha indicado, a la relación de amistad).

$\mathrm{Y}$ esto nos permite retornar al otro ejemplo de Johnson-Laird (2010) y, de este modo, entender, con la misma claridad que en [3], lo que realmente sucede en escenarios como el de [4]. Dado que una premisa implícita muy frecuente cuando se trata de relaciones es [10], la mayor parte de los individuos tiende a inferir, con base en ella, que Ann y Chris son parientes de sangre. No obstante, explicaciones adicionales sobre el carácter y la naturaleza del matrimonio como las incluidas en la experimentación de Goodwin y Johnson-Laird (2008) pueden llevar a notar que esa es una premisa errónea y que la que verdaderamente conviene a la relación de 
parentesco consanguíneo es una como [16], ya que, en tal relación, la transitividad puede ser tanto correcta como incorrecta. Así, la conclusión no sería que Ann y Chris son parientes consanguíneos, sino que pueden serlo o no, como lo demuestra esta inferencia:

[17] 1. Pab

2. $\mathrm{Pbc}$

3. $\forall x y z(P x y \wedge P y z \rightarrow P x z \vee \neg P x z)$

4. $\mathrm{Pab} \wedge \mathrm{Pbc}$

5. $\mathrm{Pab} \wedge \mathrm{Pbc} \rightarrow \mathrm{Pac} \vee \neg \mathrm{Pac}$

6. $\mathrm{Pac} \vee \neg \mathrm{Pac}$ (premisa)

(premisa)

(premisa implícita)

(Introducción de Conjunción 1, 2)

(Eliminación de Cuantificador

(Donde 'P $\alpha \beta$ ' significa aquí que $\alpha$ y $\beta$ son parientes consanguíneos, ' $a$ ' es Ann, ' $b$ ' es Beth y ' $c$ ' es Chris).

Obviamente, se puede objetar aquí que la fórmula sexta en [17] es una tautología y, por tanto, muy poco informativa. Empero, precisamente su carácter poco informativo es el que puede otorgarle su pertinencia en este caso, puesto que la naturaleza de las relaciones de parentesco es tal que no hay información suficiente con respecto a la consanguinidad, y tal situación de incertidumbre parece estar bastante bien reflejada por la mencionada fórmula. No obstante, en cualquier caso, se puede decir que tenemos, al menos, una explicación de lo que acontece y de las respuestas mayoritarias en las tareas de razonamiento con relaciones diádicas que es absolutamente coherente con los planteamientos generales de las teorías sintácticas que se pueden encontrar en la literatura especializada. De este modo, se puede decir que, si el enfoque formal no es apropiado para describir el razonamiento, la actividad inferencial y la comunicación en el ser humano, ello no es así porque no pueda resolver los problemas planteados por Johnson-Laird (2010) que han sido analizados en estas páginas. Indudablemente, si deseamos descartar ideas como la de una sintaxis del pensamiento, necesitamos mayores evidencias y apoyos empíricos y teóricos. 


\section{CONCLUSIONES}

Probablemente, el mayor problema de las teorías sintácticas no es intrínseco, sino de una índole más sociológica. Con frecuencia, las propuestas de las teorías formales no son entendidas correctamente y se producen un número considerable de malinterpretaciones. Esto es así porque, en demasiadas ocasiones, se piensa que lo que proponen es que en la mente humana solamente opera la sintaxis, sin haber espacio alguno ni para la semántica ni para la pragmática. Como se ha mostrado en las páginas precedentes, esto no es en absoluto de este modo. A pesar de su nombre, no solo la sintaxis tiene cabida en las teorías sintácticas. Otras dimensiones son posibles y los teóricos de estos enfoques, aunque habitualmente hacen especiales alusiones únicamente a la pragmática, a menudo reconocen explícitamente este punto.

Es evidente, por lo demás, que los argumentos expuestos en este trabajo muestran la estrecha correlación que existe entre las tres dimensiones. Es verdad que hemos hablado de premisas implícitas caracterizadas fundamentalmente por su forma, con estructuras como las de las fórmulas de la lógica estándar y que, por ello, remiten claramente a factores sintácticos. Sin embargo, tales premisas son reconocidas, detectadas o identificadas en contextos pragmáticos y, para llegar a ellas, como igualmente se ha mostrado, es ineludible comprender el significado exacto de la relación establecida, lo cual, indiscutiblemente, nos aboca a una dimensión semántica.

En este sentido, parece que lo apropiado es continuar en la línea de trabajos como, por ejemplo, los de López-Astorga $(2015,2016)$, en los que se apunta como posibilidad a la idea de que suceda en el pensamiento lo mismo que en el lenguaje y de que, por ende, las tres dimensiones mencionadas sean inseparables también en el primero. Así, se puede suponer que el problema reside en que tanto la visión de Johnson-Laird (2010) como la de las teorías formales son demasiado extremas. La primera tiende a ignorar y a infravalorar la sintaxis y las segundas suelen otorgarle a la semántica un segundo plano. Sin embargo, muchos trabajos en la literatura, entre ellos los de López-Astorga (2015, 2016), nos revelan que, a menudo, los resultados experimentales pueden ser explicados desde las dos perspectivas, desde una más semántica y otra más sintáctica, lo que, como LópezAstorga $(2015,2016)$ plantea, permite especular sobre la acción conjunta de ambas dimensiones en la actividad intelectual.

Claramente, la pragmática no genera ninguna dificultad, pues, como se indica también en trabajos como los mencionados, prácticamente ningún enfoque 
contemporáneo olvida su valor. Por consiguiente y ante todo esto, quizás deberíamos ir considerando la necesidad de profundizar en la idea de O'Brien (1998) señalada más arriba. Tal idea es la relativa a la no exigencia de exclusividad y a que las tesis de varias teorías pueden ser válidas al mismo tiempo. De esta manera, podríamos ir conformando una teoría global del razonamiento que integrara a la semántica, la sintaxis y la pragmática como tres aspectos esenciales de la dinámica intelectiva, sin tener ninguno ningún predominio especial sobre los demás en ningún ámbito en particular.

En cualquier caso, parece que tenemos tres posibilidades. Una sería esta última, la cual coincide con posiciones semejantes a las de López-Astorga (2015, 2016). Otra sería la de privilegiar la semántica. Por último, la tercera sería la de las teorías formales y su inclinación a la sintaxis. Estudios como el presentado aquí parecen reflejarnos que no es inadecuado decantarse por esta última posibilidad. No obstante, los vínculos obvios de las teorías formales con la semántica y la pragmática sugieren que la primera tampoco es desdeñable y que es probable que la investigación en el área nos vaya arrojando más luz conforme se vaya avanzando en mayor medida en estas temáticas.

\section{REFERENCIAS}

Braine, M. D. S., y O'Brien, D. P. (1998a). The theory of mental-propositional logic: Description and illustration. En M. D. S. Braine y D. P. O'Brien (Eds.), Mental Logic (pp. 79-89). Mahwah: Lawrence Erlbaum Associates, Inc. Publishers.

Braine, M. D. S., y O'Brien, D. P. (1998b). How to investigate mental logic and the syntax of thought. En M. D. S. Braine y D. P. O’Brien (Eds.), Mental Logic (pp. 45-61). Mahwah: Lawrence Erlbaum Associates, Inc. Publishers.

Bryant, P. E., y Trabasso, T. (1971). Transitive inferences and memory in young children. Nature, 232, 456-458.

Deaño, A. (1999). Introducción a la lógica formal. Madrid, España: Alianza Editorial.

Gamut, L. T. F. (2002). Introducción a la lógica. Buenos Aires, Argentina: Eudeba.

Gamut, L. T. F. (2010). Lógica, Lenguaje y Significado. Buenos Aires, Argentina: Eudeba.

Gentzen, G. (1934). Untersuchungen über das logische Schließen I. Mathematische Zeitschrift, 39(2), 176-210.

Gentzen, G. (1935). Untersuchungen über das logische Schließen II. Mathematische Zeitschrift, 39(3), 405-431. 
Goodwin, G. P., y Johnson-Laird, P. N. (2008). Transitive and pseudo-transitive inferences. Cognition, 108(2), 320-352.

Grice, H. P. (1975). Logic and Conversation. En P. Cole y J. L. Morgan (Eds.), Syntax and Semantics, Vol. 3, Speech Acts (pp. 41-58). New York: Academic Press.

Johnson-Laird, P. N. (2010). Against logical form. Psychologica Belgica, 50(3/4), 193221. doi: http://doi.org/10.5334/pb-50-3-4-193

Johnson-Laird, P. N., Khemlani, S., y Goodwin, G. P. (2015). Logic, probability, and human reasoning. Trends in Cognitive Sciences, 19(4), 201-214. doi: https://doi.org/10.1016/j.tics.2015.02.006

López-Astorga, M. (2015). The disjunction introduction rule: Syntactic and semantic considerations. Pragmalingüística, (23), 141-149. doi: http://dx.doi.org/10.25267/Pragmalinguistica.2015.i23.08

López-Astorga, M. (2016). Some arguments that the mental logic theory needs to clarify to continue being an alternative to the mental models theory. Civilizar, 16(31), 235-248. doi: http://dx.doi.org/10.22518/16578953.652

O'Brien, D. P. (1998). Mental logic and irrationality: We can put a man on the moon so why can't we solve those logical reasoning problems? En M. D. S. Braine y D. P. O’Brien (Eds.), Mental Logic (pp. 23-43). Mahwah: Lawrence Erlbaum Associates, Inc. Publishers.

Politzer, G. (2004). Reasoning, judgement and pragmatics. En I. A. Noveck y D. Sperber (Eds.), Experimental Pragmatics (pp. 94-115). Hampshire: Macmillan.

Tsal, Y. (1977). Symmetry and transitivity assumptions about a nonspecified logical relation. Quarterly Journal of Experimental Psychology, 29(4), 677-684. doi: https://doi.org/10.1080/14640747708400641 


\section{SOBRE EL AUTOR}

\section{Miguel López-Astorga}

Doctor en Lógica y Filosofía de la Ciencia e investigador y profesor asociado en el Instituto de Estudios Humanísticos "Juan Ignacio Molina" de la Universidad de Talca, Chile. Sus áreas de docencia e investigación son la epistemología, la lógica y la filosofía del Lenguaje.

Correo electrónico: milopez@utalca.cl 